

Annals of DAAAM for 2012 \& Proceedings of the 23rd International DAAAM Symposium, Volume 23, No.1, ISSN 2304-1382 ISBN 978-3-901509-91-9, CDROM version, Ed. B. Katalinic, Published by DAAAM International, Vienna, Austria, EU, 2012 Make Harmony between Technology and Nature, and Your Mind will Fly Free as a Bird Annals \& Proceedings of DAAAM International 2012

\title{
SINGLE-PIXEL CAMERA - SIMULATION TOOL AND THE FIRST RESULTS OF ANALYSIS OF IMAGE QUALITY
}

\author{
KOWALSKI, M[ARCIN]; PISZCZEK, M[arek]; \\ SZUSTAKOWSKI, M[ieczyslaw] \& CIURAPINSKI, W[ieslaw]
}

\begin{abstract}
The present trend of signal processing maintains that according to the Nyquist-Shannon theory a signal must be sampled at a rate at least twice its highest frequency in order to be represented without error. However, it is very common to compress the data just after sensing. This approach is very popular in the field of image acquisition. We compress images registered by a camera trading off signal representation complexity. Thus some of the information is simply thrown away.

Sparse signal approximations have become a new tool in signal processing with wide range of applications. Recently, many algorithms for signal reconstruction have been developed, however, all of them need many parameters to be properly set before using. Setting proper parameters is crucial for preparing a real model of the single pixel camera as well as for fast and efficient image synthesis. Because of high complexity of image recovery algorithms based on compressed sensing method, image synthesis process needs to be optimized.

Simulation results include quality parameters values of MSE, PSNR and SSIM and image reconstruction time. Integrated test environment can be used during the process of hardware selection as well as during camera tests with real signals.

Keywords: single pixel camera, compressed sensing, multispectral imaging
\end{abstract}

\section{INTRODUCTION}

Compressed sensing (CS) [1] is a signal processing method based on the fact that an undersampled sparse signal can be reconstructed from a small number of measurements. Images fully satisfy all the conditions needed to use compressed sensing, thus it was proved that a camera using CS and a single pixel detector can be build $[2,3]$. The camera registers random projections of a scene. The CS method shows that a signal can be compressed during acquisition and still contain nearly all useful information. The compression means that the single pixel camera registers much less information than the traditional one during the acquisition process.

From a hardware point of view the camera uses an optical modulator (micromirror array or LCD matrix) to optically calculate linear projections of the scene onto pseudorandom binary patterns. The key feature of the CS method is the ability to obtain an image with a single pixel detector by measuring the scene fewer times than the number of pixels. Evolution of the compressed sensing method and single-pixel camera has led to the development of new signal processing hardware and mathematical algorithms. Because of the high complexity of the image recovery algorithms based on the compressed sensing method, the image synthesis process needs to be optimized. In this case, the optimization means both optimization of image reconstruction accuracy and the efficiency of hardware image recovery.

Optimization of signal acquisition and processing parameters can be achieved running various camera simulations. A versatile simulation tool for image synthesis of compressed sensing based camera was prepared to simplify and gain the imaging process. The prepared test environment makes it possible to run two kinds of test - simulation of image acquisition parameters (e.g. various bit resolutions) or simulation of image geometric transformations (e.g. rotation). The basic function of this tool is an image recovery.

One of the simulator capabilities is proper image quality assessment. Because of the lack of one unified and versatile image quality metric, the simulation tool is able to compute the three most accurate [4] metrics mean squared error, peak signal-to-noise ratio, and structural similarity index.

The CS simulation tool can be used during the process of hardware selection as well as during camera tests with real signals. All tests can be run with a various number of iterations and for various image resolutions.

\section{QUALITY ASSESMENT}

Describing a recovered image is not a simple task, especially when one tries to assess the image quality from the point of view of a human eye. There are very popular image quality metrics used mainly for comparing images such as mean squared error (MSE) and peak signal-to-noise ratio (PSNR).

MSE is a function corresponding to the expected value of the squared error loss or quadratic loss, it is computed using following equation (1):

$$
M S E=\frac{1}{N \cdot M} \sum_{i=1}^{N} \sum_{j=1}^{M}\left(\left[f(i, j)-f^{\prime}(i, j)\right]^{2}\right)
$$

The PSNR is a logarithmic index, the ratio between the maximum possible power of a signal and the power of corrupting noise that affects the fidelity of its representation. The PSNR can be computed using MSE from the following equation (2):

$$
P S N R=10 \cdot \log _{10} \frac{k^{2}}{M S E}
$$






Fig. 1. Reconstruction of 8-bit monochromatic image a) $10 \%$, b) $20 \%$, c) $30 \%$, d) $40 \%$, e) $50 \%$, f) $60 \%$, g) $70 \%$, h) $80 \%$, i) $90 \%$, j) $100 \%$ iterations.

In case of using them with $\mathrm{CS}$ - recovered images can be used for describing tendencies of quality. Assessing perceptual image quality is also connected with quantifying the visibility of differences between a distorted image and an original (reference) image. One very good metric for perceptual image quality assessment is structural similarity index (SSIM) [5] based on the degradation of structural information. SSIM can be computed using the following equation (3):

$$
\operatorname{SSIM}(x, y)=\frac{\left(2 \mu_{x} \mu_{y}+c_{1}\right)\left(2 \sigma_{x y}+c_{2}\right)}{\left(\mu_{x}^{2}+\mu_{x}^{2}+c_{1}\right)\left(\sigma_{x}^{2}+\sigma_{y}^{2}+c_{2}\right)}
$$

This index gives numerical information with values from -1 to 1 and value 1 is only achievable in the case of two identical sets of data.

\section{APPLICATION}

The theory of compressed sensing was basically described in [1]. Suppose we have available undersampled data about $f$ of the following form (4):

$$
y=\Phi \cdot f
$$

One would recover $f$ by finding - among all coefficient sequences consistent with the data - the decomposition with minimum $\ell_{1}$-norm [1] (5):

$$
\min \|\tilde{x}\|_{\ell_{1}} \text { such that } \Phi^{\prime} \tilde{x}=y
$$

From an application point of view, the $\ell_{1}$ - norm minimization can be achieved using e.g. a conjugate gradient algorithm or gradient projection method. The compressed sensing simulation tool was originally designed and implemented as a script application using gradient projection method or conjugate gradient method. The basic functionality of the tool is an image recovery. The process of 8-bit image recovery with iterations from $10 \%$ to $100 \%$ is shown on Fig. 1 .

Values of image quality assessment metrics (SSIM and PSNR) for every iteration are shown on Fig. 2. It can be noticed that the SSIM index takes values from very low 0.18 up to very high 0.95 . The highest value is very close to 1 , which means that the image reconstructed from $100 \%$ of measurements is nearly the same with the original one. The PSNR unlike the SSIM shows values fluctuations.

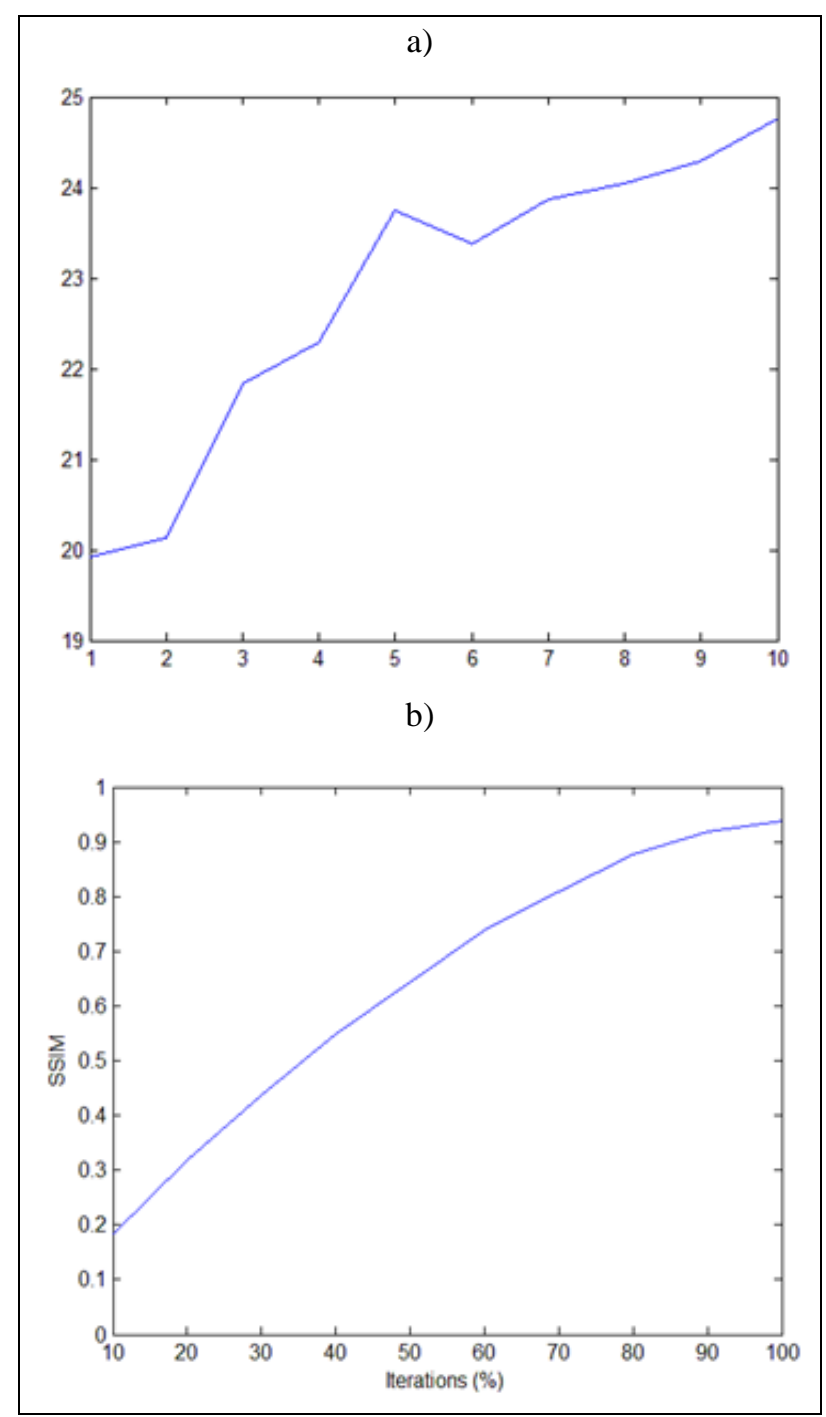

Fig. 2. Values of image quality indexes a) SSIM, b) PSNR.

\section{GEOMETRIC TRANSFORMATIONS}

Geometric transformations of images are considered as a rearrangement of the pixels in the image, particularly the image rotation. For the test purposes, multiples of $90^{\circ}$ were taken as a rotation angle. 


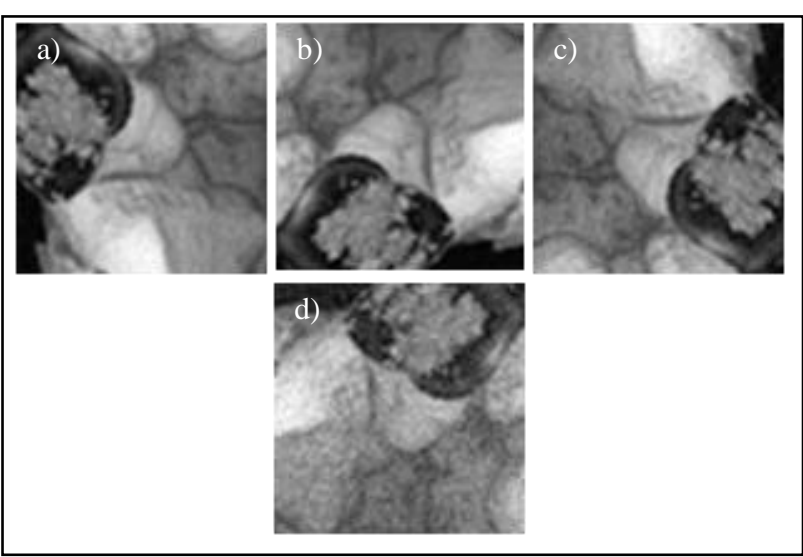

Fig. 3. Rotated images a) 0 degrees, b) 90 degrees, c) 180 degrees, d) 270 degrees, recovered with $100 \%$ iterations.

Being able to recover a geometrically transformed image using the CS method is crucial for all the applications where the image may be misaligned.

Every recovered image can be stored as a 24-bit bitmap file or as a MAT file. Rotated MRI images recovered (image resolution $64 \times 64$ pixels) with $100 \%$ of iterations using the simulation tool are presented on Fig. 3. Image quality metrics are computed for every image. SSIM values for rotated images are presented on Fig. 4.

\section{PARAMETERS OF SIGNAL ACQUISITION}

The process of building a camera is preceded by a set of decisions that have to be made very precisely. Most of these decisions consider parameters of hardware such as analog-to-digital converters or number of detectors. Because a camera based on compressed sensing registers images with single detector, the problem of matrix resolution is not relevant. Parameters of analog-to-digital converters, such as bit resolution are crucial, especially when the camera is about to acquire minimum amount of data. The simulation tool offers the possibility to recover images with data sampled with chosen bit resolution (e.g. 6 bit resolution).

During the simulations six different possibilities were tested $(4,6,8,10,12,16$ bit). Sample images reconstructed from 4-bit and 6-bit resolution data are presented on Fig. 5 and Fig. 6.

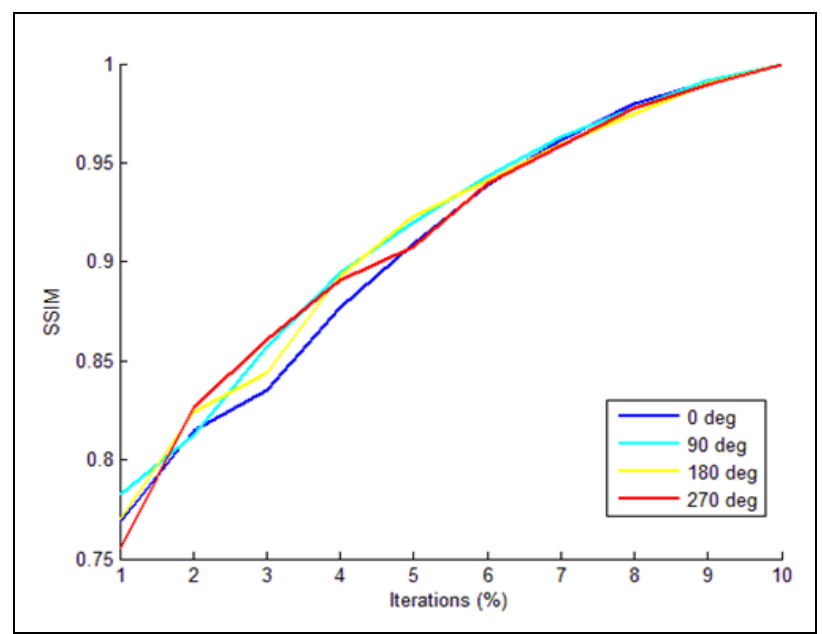

Fig. 4. SSIM values of 8bit rotated images.

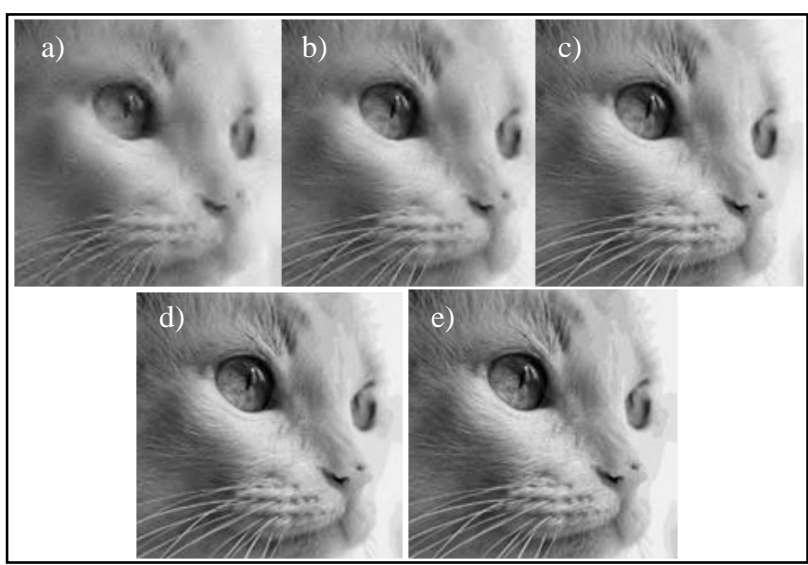

Fig. 5. Reconstruction with 4-bit data a) $10 \%$, b) $20 \%$, c) $40 \%$, d) $70 \%$, e) $100 \%$ of iterations.

According to the simulation results and the quality metrics values, the image recovery can be done almost as well with 4-bit resolution as with 16-bit (original image).



Fig. 6. Reconstruction with 6-bit data a) $10 \%$, b) $20 \%$, c) $40 \%$, d) $70 \%$, e) $100 \%$ of iterations

Images recovered with CS method can be assessed by human eye of by mathematical metric. CS recovered images should be assessed by perceptual metric, because the possible loss of data always mean lower values of traditional metrics like PSNR or MSE. This is the reason why SSIM index is used to evaluate images recovered with CS.

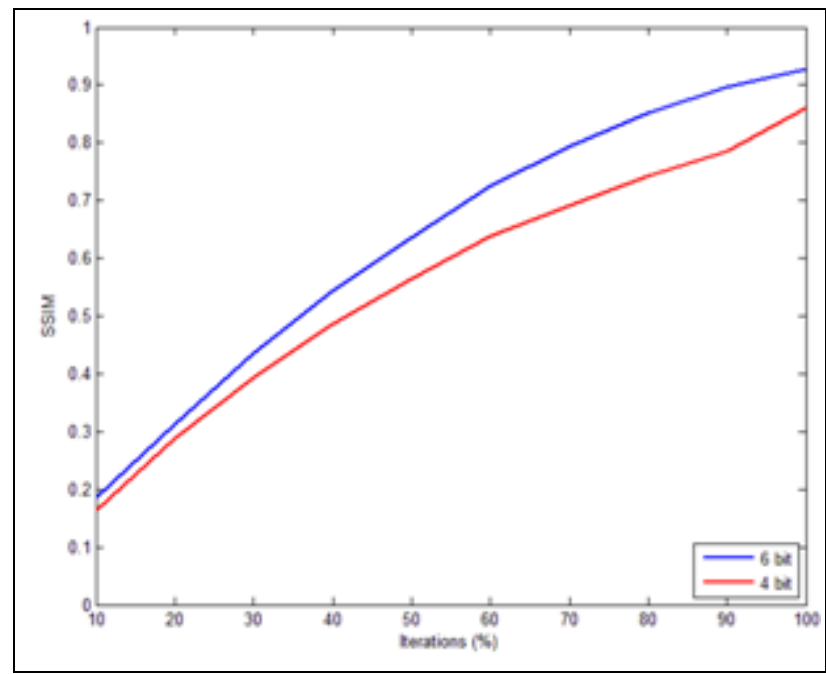

Fig. 7. Values of SSIM for 4-bit and 6-bit reconstructed image. 


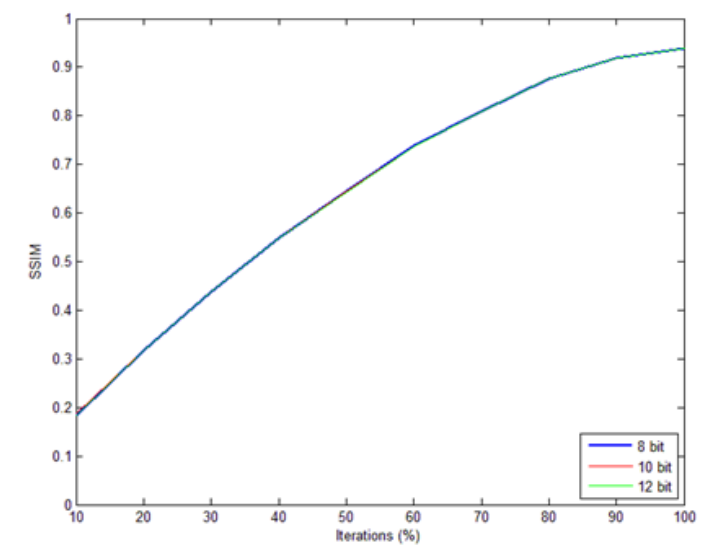

Fig. 8. Values of SSIM for 8-bit, 10-bit and 12-bit reconstructed image.

Diagrams of SSIM values for every iterations are presented on Fig. 7. One can noticed that SSIM values for 4-bit data are in the range between 0.17 and 0.85 . The same results for 6-bit data are respectively bigger, between 0.19 to 0.93 . The difference is noticeable nevertheless quality loss can be compensated by registering more measurements.

The differences between SSIM values for 8, 10 and 12 bit data are almost unnoticeable (Fig. 8).

The results presented above are the first step for building an efficient single-pixel camera working with non-expensive elements. The future research will lead to experimental confirmation of presented results. If the hardware tests confirm the simulation results it will mean that CS-based cameras can be built with non-expensive elements (low bit resolution ADCs are relatively not expensive). This also means that there are new application possibilities for the single-pixel cameras.

\section{SPECTRAL IMAGES}

Most of the CS applications consider grayscale images acquisition. The compressed sensing - based camera can be used to acquire coloured images e.g. RGB or multispectral. This can be achieved by using multiple spectral filters with only one single-pixel detector or by using a few low band single-pixel detectors without filters. The reconstruction of colour images as well as multispectral images can be the topic for further algorithm development.

\section{SUMMARY}

Compressed sensing is a very complex algorithm $[9,10]$. Its possibilities are enormous thus need to be investigated. Computer simulation is the first stage of building a single pixel camera based on compressed sensing. Before applying CS in hardware, simulation of various possibilities need to be run. This was the reason for building a simulation tool which can be used to reconstruct images with various geometric transformations, various noise types with selected levels or with different bit resolution. Resulting images can be automatically evaluated by three most accurate image quality metrics.
Simulation results can be helpful in the process of hardware selection. Recent results give a good perspective of using low bit resolution (even 4 bit) analogue-to-digital converters.

The recent work in the field of compressive sensing enabled effective image reconstruction from a subset of the measurements. The software architecture is flexible and extensible.

The paper described a flexible software platform which we are developing for dynamically capturing and registering images.

Another step in the research process is building a hardware test environment of the compressed sensing based camera. This means selecting the most apropriate hardware and confirming the computer simulation results with laboratory equipment.

The simulation tool will allow to define a noise level and the type of noise (with uniform or normal distribution). By adding generated noise to an image we will simulate the cumulative image noise and test the algorithm ability to reconstruct the image without noise.

\section{ACKNOWLEDGEMENTS}

This article was prepared based on development project realized in $12^{\mathrm{th}}$ competition organized by Ministry of Science and Higher Education - "Integrated Laser Photography System for open space monitoring and terrorist threats prevention", OR00000312.

\section{REFERENCES}

[1] Donoho D., "Compressed sensing", IEEE Trans. on Information Theory, 52(4), pp. 1289 - 1306, April 2006

[2] Takhar, D., Laska, J., Wakin, M., Duarte, M., Baron, D., Sarvotham, S., Kelly, K., Baraniuk, R., "A New Compressive Imaging Camera Architecture using Optical-Domain Compression", Proc. of Computational Imaging IV at SPIE Electronic Imaging, San Jose, CA, Jan. 2006

[3] Duarte, M.F., Davenport, M. A., Takhar, D., Laska, J.N., Sun, T., Kelly, K.F., Baraniuk, R., "Single Pixel Imaging via Compressive Sampling", IEEE Signal Processing Magazine, March 2008

[4] Wang, Z., Bovik, A.C., Sheikh, H. R., Simoncelli, E. P., "Image Quality Assessment: From Error Visibility to Structural Similarity", IEEE transactions on image processing, vol. 13, no. 4, april 2004

[5] Wang, Z., Li, Q., "Information content weighting for perceptual image quality assessment", IEEE Transactions on Image Processing, vol. 20, no 5, pp. 1185-1198, May 2011

[6] Baraniuk, R., Cevher, V., Duarte, M., Hegde, C., "Model-based Compressive Sensing", IEEE Transactions In Information Theory, Vol. 56, pp 1982-2001 (2010)

[7] Golub, G. H.; Van Loan, Ch. F., "Matrix computations" (3rd ed.), Johns Hopkins University Press, Chapter 10, ISBN 0-8018-54148

[8] Li, C.; Sun, T.; Kelly, K. F.; Zhang, Y.; "A Compressive Sensing and Unmixing Scheme for Hyperspectral Data Processing", IEEE, September 2011

[9] E. Cand es, M. B. Wakin, and S. Boyd, "Enhancing sparsity by reweighted $\ell 1$ minimization," J. Fourier Analysis and Appl., vol. 14,no. 5, pp. 877-905, 2008.

[10] J. Romberg, "Compressive sensing by random convolution," SIAM J. Imaging Sci., vol. 2, no. 4, pp. 1098-1128, 2009 